\title{
Multimodal imaging of buried optic nerve head drusen
}

\author{
Delia D. Wang ${ }^{1} \cdot$ James C. Y. Leong ${ }^{1} \cdot$ Jesse Gale ${ }^{2,3} \cdot$ Anthony P. Wells ${ }^{2,3}$
}

Received: 7 December 2016 / Accepted: 13 December 2017 / Published online: 30 January 2018

(c) The Royal College of Ophthalmologists 2018

\section{Introduction}

The literature on the appearance of buried optic nerve head drusen (ONHD) on spectral domain optical coherence tomography (SD-OCT) is conflicting. Some authors have described a focal subretinal hyperreflective mass posterior to the outer plexiform and outer nuclear layers [1]; whereas others described ONHD as a hyporeflective round or ovoid structure containing hyperreflective foci, located anterior to the lamina cribrosa [2-4]. In this study, we aimed to clarify this controversy with multimodal imaging. We attempted to correlate our findings with published histological findings.

\section{Methods}

This retrospective, observational study assessed five consecutive patients referred by optometrists to an acute ophthalmology service with suspected swollen optic discs. The mean age was 28 years (range: 13-49 years). The presence of buried ONHD was diagnosed clinically with history and dilated fundoscopy and confirmed with ultrasonography. None of the patients had exposed or visible ONHD. All patients underwent imaging with colour fundus photography, fundus autofluorescence and SD-OCT (Spectralis, Heidelberg Engineering, Germany). We obtained 49 horizontal enhanced depth imaging SD-OCT scans in a square raster pattern centred on the optic nerve head of each eye. Each horizontal scan consisted of at least 12 OCT B-scans, which were averaged using active retinal tracking. This scan protocol enabled highresolution visualisation of deeper structures within and around the optic nerve head.

Delia D. Wang

Drdelia.wang@gmail.com

1 Ophthalmology Department, Christchurch Hospital, Christchurch, New Zealand

2 Capital Eye Specialists, Wellington, New Zealand

3 University of Otago, Wellington, New Zealand

\section{Results}

In point-by-point comparison of the multimodal imaging, we found both hyperreflective and smaller hyporeflective structures on SD-OCT (Fig. 1). The larger peripapillary hyperreflective structures did not demonstrate autofluorescence, whereas areas of autofluorescence appeared to localise to the smaller hyporeflective structures located anterior to the lamina cribrosa.

\section{Discussion}

Histologically, ONHD have been described as globular aggregates of concentrically laminated calcified material, just anterior to the lamina cribrosa [5]. Electron microscopy revealed aggregates of extracellular calcified mitochondria, presumed to have been extruded from disrupted axons. It was hypothesised that a narrow scleral canal leads to axoplasmic stasis, leading to degenerative extrusion of calcified mitochondria, providing a nidus for further calcium deposition and drusen formation [5].

We hypothesise that the larger hyperreflective structures seen on SD-OCT represent obstructed axoplasmic material; whereas the smaller hyporeflective, autofluorescent structures represent calcified ONHD, comprising degenerated extracellular mitochondria. As suggested by Sato and colleagues, mitochondria contain fluorescent porphyrins, whereas other examples of axoplasmic stasis in the retinal ganglion cells (cotton wool spots, papilloedema) do not have hyporeflective foci or autofluorescence [2].

Correlation between different imaging modalities raises a fundamental issue of definition. Here, we have correlated the hyporeflective structures with autofluorescence, but neither OCT nor autofluorescence are required to define or diagnose ONHD. As imaging of increasing resolution and penetration is developed, it is likely that we will detect ONHD that are too small for detection on clinical examination or ultrasonography. In some cases, these findings will be of clinical relevance and in others, not. Prospective observation will allow the long-term prognostic importance of these findings to be evaluated. Such observations might 

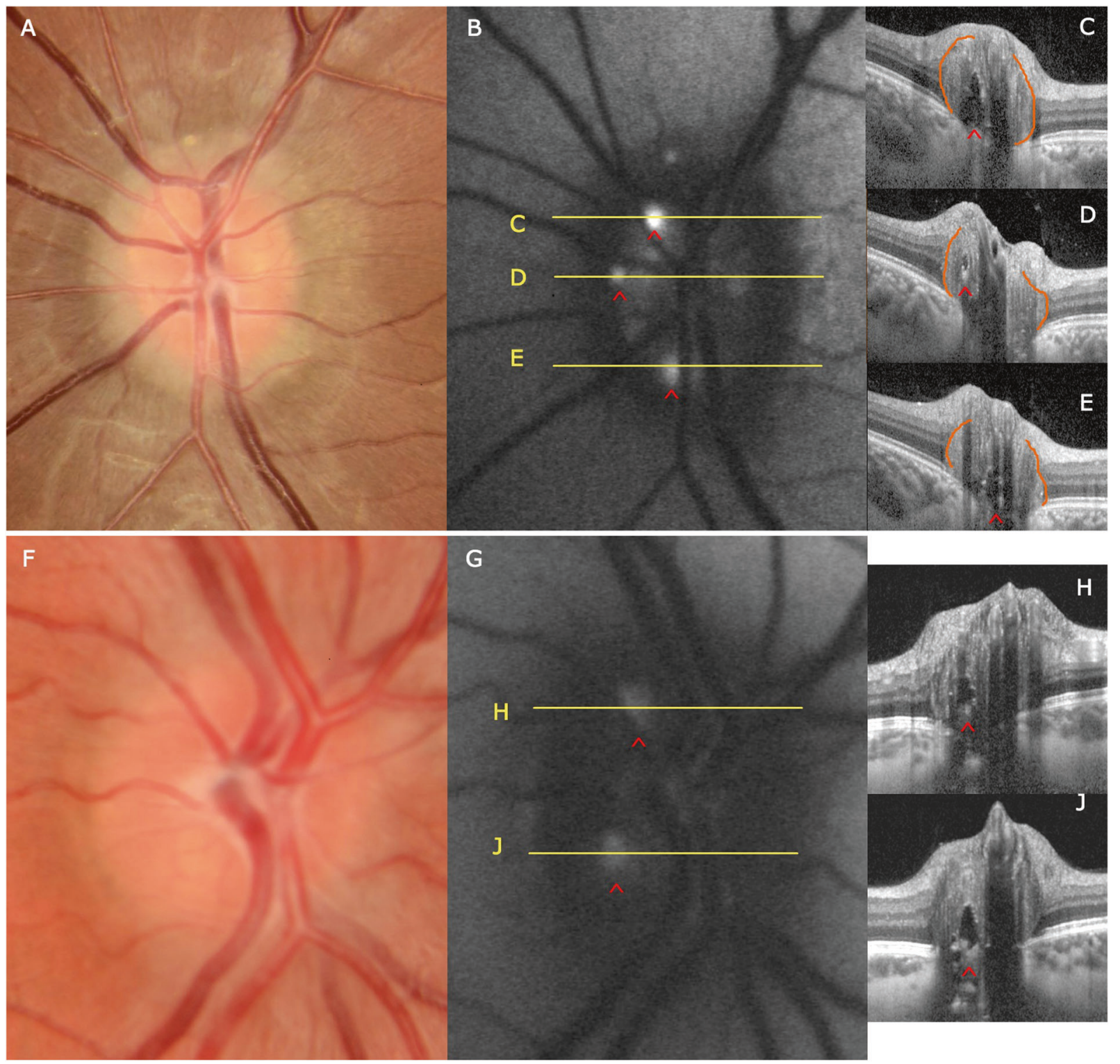

Fig. 1 Multimodal imaging comprising colour fundus photographs (a and $\mathbf{f}$ ), fundus autofluorescence images and spectral domain optical coherence tomography (SD-OCT) from two patients in our series with buried optic nerve head drusen (ONHD). The autofluorescent ONHD (b and g) appeared as small hyporeflective round structures with

facilitate the study of interventions by offering a clinically meaningful biomarker.

\section{Compliance with ethical standards}

Conflict of interest The authors declare that they have no competing interests.

\section{References}

1. Lee KM, Woo SJ, Hwang J-M. Morphologic characteristics of optic nerve head drusen on spectral-domain optical coherence tomography. Am J Ophthalmol. 2013;155(6):1139-47.e1. hyperreflective foci, anterior to lamina cribrosa on SD-OCT (c-e and $\mathbf{h}-\mathbf{j}$; marked with ${ }^{\wedge}$ ). Larger hyperreflective peripapillary subretinal egg-shaped structures were also observed on SD-OCT, which we hypothesise to represent obstructed axoplasmic material (c-e; margins outlined in orange, and $\mathbf{h}-\mathbf{j}$; not outlined)

2. Sato T, Mrejen S, Spaide RF. Multimodal imaging of optic disc drusen. Am J Ophthalmol. 2013;156(2): 275-82.e1.

3. Silverman AL, Tatham AJ, Medeiros FA, Weinreb RN. Assessment of optic nerve head drusen using enhanced depth imaging and swept source otical coherence tomography. J Neuro-Ophthalmol. 2014;34(2):198-205.

4. Merchant KY, Su D, Park SC, Qayum S, Banik R, Liebmann JM, et al. Enhanced depth imaging optical coherence tomography of optic nerve head drusen. Ophthalmology. 2013;120 (7):1409-14.

5. Auw-Haedrich C, Staubach F, Witschel H. Optic disk drusen. Surv Ophthalmol. 2002;47(6):515-32. 